\title{
PRIMITIVE IDEALS IN VON NEUMANN ALGEBRAS ${ }^{1}$
}

\author{
HERBERT HALPERN
}

\begin{abstract}
For a von Neumann algebra it is shown that the set of primitive ideals containing a fixed maximal ideal of the center is sequentially closed in the order topology defined on the set of all ideals containing the maximal ideal. As a corollary, it is shown that every ideal generated by a sequence of elements of a von Neumann algebra and a maximal ideal of the center is either primitive or simple modulo a primitive ideal.
\end{abstract}

1. Preliminaries. Let $A$ be a von Neumann algebra with center $Z$. Let $\zeta$ be a maximal ideal of $Z$ and let $C_{\zeta}$ be the set of all (closed twosided) ideals of $A$ containing $\zeta$. The set $C_{\zeta}$ is linearly ordered by inclusion and therefore every ideal $I$ in $C_{\zeta}$ is prime, i.e. $I \supset I^{\prime} I^{\prime \prime}$ implies $I \supset I^{\prime}$ or $I \supset I^{\prime \prime}$. The set of all prime ideals of $A$ is $\bigcup_{\zeta} C_{\zeta}$. It is known that every primitive ideal, i.e. the kernel of an irreducible representation of $A$, is prime. Since every prime ideal is primitive for a separable $C^{*}$-algebra [1], the question arises as to whether a prime ideal is primitive for other algebras. We consider some aspects of that question in this paper.

Notation. $A$ is a von Neumann algebra with center $Z ;(S)$ is the set of projections in $S \subset A ; a, b, c$ are elements of $A ; e, f, g$ are elements of $(A)$; $p, q$ are elements of $(Z) ; \zeta$ is a maximal ideal of $Z$; [ $\zeta]$ is the ideal in $A$ generated by $\zeta$.

2. Ideals. Given $e \in(A)$, let $S(e)$ denote the set of all finite sums of orthogonal projections $e_{i}(1 \leqq i \leqq n)$ in $A$ with $e_{i}<e$.

Lемма 1. If $e$ and $f$ are projections in $A$ such that $e \in S(f)$, then $S(e) \subset S(f)$.

Proof. It is sufficient to show that $g \in S(f)$ whenever $g \leqq e$. Let $e_{i}(1 \leqq i \leqq n)$ be orthogonal projections of sum $e$ with $e_{i}<f$. The range projection $g_{i}^{\prime}$ of $g e_{i}$ satisfies the relations $g_{i}^{\prime} \leqq g$ and $g_{i}^{\prime}<e_{i}[3$, III, 1 , Proposition 2]. Setting $g_{0}^{\prime \prime}=0$ and $g_{k}^{\prime \prime}=\bigvee\left\{g_{i}^{\prime} \mid 1 \leqq i \leqq k\right\}$ for $1 \leqq k \leqq n$, we get that $\left\{g_{k}^{\prime \prime}-g_{k-1}^{\prime \prime} \mid 1 \leqq k \leqq n\right\}$ implements the relation $g \in S(f)$ since $g_{k}^{\prime \prime}-g_{k-1}^{\prime}<g_{k}^{\prime \prime}<e_{i}<f[3$, III, $\S 1$, Corollary 1, Proposition 2]. Q.E.D.

The next definition was considered in a less general case in [5].

Received by the editors October 2, 1972.

AMS (MOS) subject classifications (1970). Primary 46L10.

Key words and phrases. von Neumann algebra, primitive ideal.

1 Research supported by NSF Grant GP-33030. 
Definition 2. Let $(I(e))$ be the family of all projections $f$ in $A$ such that $p f^{\prime} \in[\zeta]$ whenever $p e<p f^{\prime}$ for any $f^{\prime} \in S(f)$ and $p \in(Z)$. Let $(J(e))$ be the family of all projections $f$ in $A$ such that $p f<p g$ for some $g \in S(e)$ and $p \in(Z) \backslash \zeta$.

We collect some statements we need in the next lemma.

LEMMA 3. The following statements are true:

(i) $(I(e))$ and $(J(e))$ are the sets of all projections of the ideals $I(e)$ and $J(e)$;

(ii) $I(e) \supset[\zeta]$;

(iii) $J(e)$ is generated by $e$ and $\zeta$;

(iv) if $e \notin[\zeta]$, then $J(e)$ is the smallest ideal of $A$ properly containing $I(e)$;

(v) the ideal $J(e)$ is of the form $J(e)=I(f)$ if e is a properly infinite projection not in [ל].

Proof. (i) A short calculation using Lemma 1 and [3, III, §1, Corollary 1, Proposition 2] shows that $(I(e))$ and $(J(e))$ are $p$-ideals in the sense of F. B. Wright [7] and hence the sets of projections of certain ideals in $A$.

(ii) and (iii) obvious.

(iv) If $f \in(I(e))$ and if $p \in(Z)$ with $p e<p f$ and $(1-p) f<(1-p) e$, then $p f \in[\zeta]$ and so $f \in J(e)$. Thus we get that $I(e) \subset J(e)$. Conversely, let $I$ be an ideal that properly contains $I(e)$. Then there is an $f \in(I)$, an $f^{\prime} \in S(f)$, and a $p \in(Z)$ with $p e<p f^{\prime}$ and $p f^{\prime} \notin[\zeta]$. Because $p f^{\prime} \in I$ and $[\zeta] \subset I$, we see that $p e \in I$ and so $J(e) \subset I$. By hypothesis, $e \notin[\zeta]$ and so $I(e) \neq J(e)$.

(v) Since the properly infinite projection $e$ can be expressed as a sum of $n(n=1,2, \cdots)$ orthogonal projections equivalent to $e$, we see that $S(e)=\{f \in(A) \mid f<e\}$. Since $S(e)$ is a $p$-ideal [7], there is an ideal $I$ in $A$ such that $(I)=S(e)$. If $\left\{a_{i}\right\}$ is a bounded subset of $I$ and $\left\{p_{i}\right\}$ a corresponding orthogonal set in $(Z)$ of sum 1 , then $\sum p_{i} a_{i}$, which converges in the strong operator topology, is in $I$. Indeed, the obvious case in which each $a_{i} \in(I)$ immediately implies the most general case. There is a properly infinite projection $f \notin[\zeta]$ and a $p \in(Z)$ such that $S(e)=\{g \in(A p) \mid q f<q g$ for some $q \in(Z)$ implies $q f=0\}$ [5, Theorem 2.4]. We show $J(e)=I(f)$. Notice that $p \notin \zeta$ since $e p=e$. If $g \in S(e)$ and if $q \in(Z)$ with $q f<q g$, then $q f=0$ and thus $q \in \zeta$. Hence $e \in I(f)$. By (ii) and (iii) we get $J(e) \subset I(f)$. Conversely, if $q$ is the largest element of $(Z)$ such that $q f<q g$ for given $g \in(I(f))$, then $q g \in[\zeta]$. We also have that $(1-q) p g \in S(e)$. Because $1-p \in[\zeta]$, we get that $g$ is in the ideal $J(e)$ by (ii) and (iii). Thus we have that $I(f) \subset J(e)$. Q.E.D.

We now consider the set $C=C_{\zeta}$ of prime ideals in $A$ that contain $\zeta$ introduced in $\S 1$. The set $C$ linearly ordered by inclusion. We consider the usual order topology on $C$. 
Theorem 4. The set $P$ of primitive ideals in $A$ that contain $\zeta$ is a sequentially closed subset of $C$.

Proof. If $\left\{I_{n}\right\}$ is a sequence in $P$ with $\lim I_{n}=I$, then $\bigvee_{m} \wedge\left\{I_{n} \mid n \geqq m\right\}$. So it is sufficient to show that any strictly increasing (resp. decreasing) sequence $\left\{I_{n}\right\}$ in $C$ has least upper (resp. greatest lower) bound in $P$.

First we consider the least upper bound. We may assume that $I_{0}=[\zeta]$. There are $e_{n}$ with $I_{n-1} \subset I\left(e_{n}\right) \subset J\left(e_{n}\right) \subset I_{n}$ by Lemma 3(iv). Because $I=\bigvee I_{n}=\bigvee I\left(e_{n}\right)$, we may assume that $I_{n}=I\left(e_{n}\right)(n \geqq 1)$. Furthermore, we easily verify that $I_{n}=I\left(\bigvee\left\{e_{m} \mid m \leqq n\right\}\right)$ and so we may assume that $e_{1} \leqq$ $e_{2} \leqq \cdots$. Since each proper ideal of $A$ is the intersection of primitive ideals [2, 2.7.3], the ideal $I_{n}(n \geqq 1)$ is primitive (Lemma 2(iv)). This means that there is a pure state $\phi_{n}$ of $A$ such that $\phi_{n}\left(e_{n}\right)=1$ and $\phi_{n}\left(I_{n}\right)=0$. Let $U$ be a free ultra-filter of the natural numbers and let $\phi$ be the state of $A$ given by $\lim _{U} \phi_{n}(a)=\phi(a)$ for all $a \in A$. We show that the canonical representation $\pi$ of $A$ induced by $\phi$ is an irreducible representation with kernel $I$.

If $\psi$ is a state of $A$, set $L(\psi)=\left\{a \in A \mid \psi\left(a^{*} a\right)=0\right\}$. To show $\pi$ is irreducible, it is sufficient to show that, given $a$ and $b$ in $A$ with $\|a-L(\phi)\|=$ $\|b-L(\phi)\|=1$ and any $\varepsilon>0$, there is a $c \in A$ with $\|c\| \leqq 1+\varepsilon$ and $c a-$ $L(\phi)=b-L(\phi)$. Given $0<\eta<1$ with $(1+\eta)(1-\eta)^{-1}<1+\varepsilon$, there is a $u \in U$ so that $\left\|a-L\left(\phi_{i}\right)\right\|$ and $\left\|b-L\left(\phi_{i}\right)\right\|$ are in the real interval [ $1-\eta$, $1+\eta]$ for all $i \in u$. Since $\phi_{i}$ is a pure state, there is a $c_{i} \in A$ with $\left\|c_{i}\right\| \leqq$ $(1+\eta)(1-\eta)^{-1} \leqq 1+\varepsilon$ such that $c_{i} a-L\left(\phi_{i}\right)=b-L\left(\phi_{i}\right)$ for $i \in u$ [6]. Now let $f_{i}$ be the range projection of $b e_{i}$ and let $g_{i}$ be the range projection of $a e_{i}$. Since $e_{i} \leqq e_{i+1}$, we have that $f_{i} \leqq f_{i+1}$ and $g_{i} \leqq g_{i+1}$ for every $i$. For convenience, let $f_{0}=g_{0}=0$. The elements $c=\sum\left\{\left(f_{i}-f_{i-1}\right) c_{i}\left(g_{i}-g_{i-1}\right) \mid i \in u\right\}$ exists in the strong operator topology and $\|c\| \leqq 1+\varepsilon$. For any $i \in u$ we get

$$
\begin{aligned}
c a-L\left(\phi_{i}\right) & =c a e_{i}-L\left(\phi_{i}\right)=c\left(g_{i}-g_{i-1}\right) a-L\left(\phi_{i}\right) \\
& =\left(f_{i}-f_{i-1}\right) c_{i}\left(g_{i}-g_{i-1}\right) a-L\left(\phi_{i}\right) \\
& =\left(f_{i}-f_{i-1}\right) b-L\left(\phi_{i}\right)=b-L\left(\phi_{i}\right) .
\end{aligned}
$$

Hence, we get $c a-L(\phi)=b-L(\phi)$. Consequently $\pi$ is irreducible.

We have that $\pi\left(e_{i}\right)=0$ for every $i$; indeed, there is a $u \in U$ with $\{1,2, \cdots, i+1\} \cap u=\varnothing$. Since $\pi$ certainly vanishes on $[\zeta]$, the kernel of $\pi$ contains $I$ (Lemma 3(iii)). Conversely, let $f \in(A) \backslash I$. We show $\pi(f) \neq 0$. Suppose we have found $p_{i} \in(Z) \backslash \zeta, f_{i} \in(f A f)$, and $v_{i} \in A$ for $1 \leqq i \leqq n$ such that the $f_{i}$ are mutually orthogonal and such that $v_{i}^{*} v_{i}=\left(e_{i}-e_{i-1}\right) p_{i}$ and $v_{i} v_{i}^{*}=f_{i}(1 \leqq i \leqq n)$. Here $e_{0}=0$. There is a maximal $p_{n+1}$ in $(Z)$ such that $\left(e_{n+1}-e_{n}\right) p_{n+1}<\left(f-\sum\left\{f_{i} \mid 1 \leqq i \leqq n\right\}\right) p_{n+1}$. We have that $p_{n+1} \notin \zeta$; otherwise, $f \in I_{n+2}$, a contradiction. There is a partial isometry $v_{n+1}$ in $A$ such that $v_{n+1} v_{n+1}^{*}=f_{n+1} \leqq f-\sum\left\{f_{i} \mid 1 \leqq i \leqq n\right\}$ and $v_{n+1}^{*} v_{n+1}=\left(e_{n+1}-e_{n}\right) p_{n+1}$. By 
induction, we may assume $p_{i}, f_{i}, v_{i}$ are defined for all $i=1,2, \cdots$. Setting $v=\sum v_{i}$ in the strong operator topology, we get that $\pi(f) v-L(\phi)=$ $v-L(\phi) \neq 0$ since $\phi_{i}\left(v^{*} v\right)=\phi_{i}\left(p_{i+1}\left(e_{i+1}-e_{i}\right) v^{*} v\left(e_{i+1}-e_{i}\right)\right)=\phi_{i}\left(e_{i+1}\right)=1$ for every $i$. This completes the proof for strictly increasing sequences.

The proof for decreasing sequences is analogous to proof for the increasing sequence. We also note that here a proof is also available from the theory of $C^{*}$-algebras using the fact that the space of primitive ideals is a Baire space with the hull-kernel topology (cf. [2, 3.4.13]). Q.E.D.

COROLlaRy 5. Let I be an ideal of $A$ generated by the sequence $\left\{a_{i}\right\}$ in $A$ and the ideal $\zeta$.

(1) Then I satisfies one of the following conditions:

(i) $I \in P$.

(ii) $I=J(e)$ for some $e \in(A)$.

(2) If I contains a properly infinite projection not in $\zeta$, then $I \in P$.

(3) If $A$ is purely infinite, then $I \in P$.

Proof. (1) The ideal $I$ is generated by $\zeta$ and the spectral projections $e_{n}(n=1,2, \cdots)$ of $\sum a_{i}^{*} a_{i} / 2^{i}\left(\left\|a_{i}\right\|^{2}+1\right)$ corresponding to the intervals $\left[n^{-1},+\infty\right)$. Thus, we find that $I=\bigvee J\left(e_{n}\right)$. If $I \neq J\left(e_{n}\right)$ for all $n$, then $I \in P$ by Theorem 4 .

(2) If $e$ is a properly infinite projection in $I \backslash[\zeta]$, then $I=J\left(e \vee e_{n}\right)$. Here, however, each $J\left(e \vee e_{n}\right)$ is primitive (Lemma 3(iii) and (iv)).

(3) If $I=[\zeta]$, then $I$ is primitive [4, Theorem 4.7]. The other case is treated in (2). Q.E.D.

\section{BIBLIOGRAPHY}

1. J. Dixmier, Sur les $C^{*}$-algebres, Bull. Soc. Math. France 88 (1960), 95-112. MR 22 \#12408.

2. ——, Les $C^{*}$-algèbres et leurs représentations, Gauthier-Villars, Paris, 1964. MR 30 \#1404.

3. - Les algèbres d'opérateurs dans l'espace Hilbertien, Gauthier-Villars, Paris, 1969.

4. H. Halpern, Irreducible module homomorphisms of a von Neumann algebra into its center, Trans. Amer. Math. Soc. 140 (1969), 195-221. MR 39 \#3322.

5. - Essential central spectrum and numerical range for elements of a von Neumann algebra, Pacific J. Math. 43 (1972), 349-380.

6. R. V. Kadison, Irreducible operator algebras, Proc. Nat. Acad. Sci. U.S.A. 43 (1957), 273-276. MR 19, 47.

7. F. B. Wright, A reduction for algebras of finite type, Ann. of Math. (2) 60 (1954), 560-570. MR 16, 375.

Department of Mathematics, University of Cincinnati, Cincinnati, Ohio 45221 